University of Nebraska - Lincoln

DigitalCommons@University of Nebraska - Lincoln

Faculty Publications - Textiles, Merchandising and Fashion Design

Textiles, Merchandising and Fashion Design,

Department of

April 2004

\title{
Improving Knit Fabric UPF Using Consumer Laundry Products: A Comparison of Results Using Two Instruments
}

\author{
Jihyun Kim \\ Virginia Polytechnic Institute and State University \\ Janis Stone \\ lowa State University \\ Patricia Crews \\ University of Nebraska, pcrews@unl.edu \\ Mack Shelley II \\ lowa State University \\ Kathryn L. Hatch \\ University of Arizona
}

Follow this and additional works at: https://digitalcommons.unl.edu/textiles_facpub

Part of the Art and Design Commons

\footnotetext{
Kim , Jihyun ; Stone , Janis ; Crews , Patricia ; Shelley, Mack II; and Hatch , Kathryn L., "Improving Knit Fabric UPF Using Consumer Laundry Products: A Comparison of Results Using Two Instruments" (2004). Faculty Publications - Textiles, Merchandising and Fashion Design. 2. https://digitalcommons.unl.edu/textiles_facpub/2

This Article is brought to you for free and open access by the Textiles, Merchandising and Fashion Design, Department of at DigitalCommons@University of Nebraska - Lincoln. It has been accepted for inclusion in Faculty Publications - Textiles, Merchandising and Fashion Design by an authorized administrator of DigitalCommons@University of Nebraska - Lincoln.
} 


\section{Improving Knit Fabric UPF Using}

Consumer Laundry Products: A

\section{Comparison of Results Using Two} Instruments

Jihyun Kim, Virginia Polytechnic Institute and State University

Janis Stone, lowa State University

Patricia Crews, University of Nebraska

Mack Shelley II, lowa State University

Kathryn L. Hatch, University of Arizona

Abstract: This study investigated the use of two instruments to measure the ultraviolet protective factor (UPF) of T-shirt knit fabrics. After various laundering treatments, specimens were cut and UPF was measured from the wale, course, and bias directions with the ISO MET $^{\circledR}$ UV-Meter and the Cary UV-Visible Spectrophotometer. Similar results were found between the two instruments and among repeated measures. Before UPF measurement, the shirt fabrics were repeatedly laundered using various household detergents and laundering additives commonly available to consumers. Statistically significant effects on the mean UPF values were found related to type of fabric, type of detergent/laundry additives, number of repeated launderings, and interaction effects of combinations of the three factors. Consumers can improve the UPF of their cotton and cotton-blend clothing by

From Family and Consumer Sciences Research Journal, Vol.

33, No. 2, December 2004, pp. 141-158

DOI: $10.1177 / 1077727 \times 04269185$

(C) 2004 American Association of Family and Consumer

Sciences using detergents with brightener and/or products containing ultraviolet absorbers. Conclusions and implications for educators and apparel and textiles industry are provided.

Keywords: ultraviolet radiation; knit fabrics; laundering; UV-absorbers; optical brighteners

The ultraviolet protective factor (UPF) has been introduced for rating and comparison of fabrics with regard to sun protection. Recently, the American Society for Testing and Materials (ASTM) issued a standard, ASTM D 6603, which provides clothing manufacturers a guide to labeling fabrics that have been tested to determine their transmission of ultraviolet radiation (UVR) of the sun and UPF (ASTM, 2003). As this labeling standard has become available, clothing suppliers have begun using UPF as an advertising appeal (L. L. Bean, Inc., 2003). In some cases, this merchandise is priced far above that of comparable merchandise that does not carry a UPF rating, making it appear costly to many consumers.

The effect of various textile properties on UVR transmission, fabric UPF, and the protection tested fabrics might provide is well documented in the literature. Research reports that provide summaries of this literature are Capjack et al. (1994);Crews, Kachman, and Beyer (1999); Davis, Capjack, Kerr, and Fedosejevs (1997); and Stanford, Georgouras, and Pailthorpe (1995a).

Previous research also has demonstrated that dyes, ultraviolet absorbers, laundering with American Association of Textile Chemists and Colorists (AATCC) detergent, and repeated launderings can improve fabric UPF (Davis et al., 1997; Eck- 
hardt \& Rohwer, 2000; Stanford et al., 1995a, 1995b; Wang et al., 2001; Zhou \&Crews, 1998). The AATCC detergent is a standard detergent used in AATCC laundering tests. However, no study was identified that investigated whether these effects could be obtained using commercial detergents available to consumers. Furthermore, no study was identified that compared the results of using two different UPF measuring instruments to obtain UPF values of specimens.

Thus, a primary purpose of this investigation was to determine whether similar UPF results would be obtained using an ISO-MET ${ }^{\circledR}$ UV-Meter and a Cary UV Visible Spectrophotometer. Hereafter, these instruments will be referred to as the UVM and SPM, respectively. The latter is broadly accepted as the instrument of choice for UPF measurement. The UVM is a small, portable instrument that offers a convenient means of demonstrating fabric UPF differences in digital format. If found to provide reliable results, this instrument would have great benefit for garment producers, retailers, educators, and others wishing to show fabric UPF differences to customers or students. A secondary purpose was to demonstrate that home laundering processes using household laundering products could raise UPF levels of low-cost, knit T-shirts above the UPF 5 that Wang et al. (2001) reported from tests of cotton T-shirts before laundering. If so, this would provide a low-cost alternative for consumers compared with the purchase of garments sold with a claim of UV protective capability, usually high-cost garments.

Specifically, because the SPM requires measurement of specimens from three different measuring directions, the purposes of this study were to determine (a) whether there were significant instrument-related differences in UPF results based on observations from three different specimen measuring directions (i.e., wales, courses, bias), (b) whether there were differences in the UPF values found in cotton jersey and blended pique-stitch knit fabrics, (c) the effects of detergents and laundering additives available on the consumer market on the UPF values of the knit fabrics, and (d) the effects of repeated laundering treatments on those UPF values.

\section{REVIEW OF LITERATURE}

\section{Ultraviolet Protection Factor Definition and Measurement}

UPF has been widely adopted and currently is used to describe the level of UVR protection provided by textile fabrics (Eckhardt \& Rohwer, 2000; Pailthorpe, 1998; Srinivasan \& Gatewood, 2000; Stanford et al., 1995a, 1995b; Wang et al., 2001; Zhou \& Crews, 1998). The meaning of UPF to consumers is interpreted in the same way as sun protective factor (SPF) used for sunscreens, with higher values representing increasing protection levels.

Three U.S. Standard documents describe the process for preparing, testing, and labeling fabric as being UV-protective. ASTM D6544, Standard Practice for Preparation of Textiles Prior to Ultraviolet (UV) Transmission Testing, defines the standardized exposures to laundering, simulated sunlight, and chlorinated pool water that cloth, labeled as UV-protective, must be exposed to before testing for UVR transmission (ASTM, 2001 a).

Second, the AATCC Test Method 183-2000 requires use of an SPM, or spectroradiometer, that measures transmission of UVR at known wavelength intervals (AATCC, 2001). These instruments are fitted with software that computes the UPF value 
as well as the transmitted UV-alpha (UV-A) and UV-beta (UVB). This AATCC Test Method 183-2000 computes UPF as "the ratio of the erythemally weighted UVR irradiance at the detector with no specimen to the erythemally weighted UVR irradiance at the detector with a specimen [fabric] present" (AATCC, 2001, p. 349). Under this definition, UPF is calculated using Equation 1:

$$
\mathrm{UPF}=\frac{\sum_{280 \mathrm{~nm}}^{400 \mathrm{~nm}} \mathrm{E}_{\lambda} * \mathrm{~S}_{\lambda} * \Delta \lambda}{\sum_{280 \mathrm{~nm}}^{400 n m} \mathrm{E}_{\lambda} * \mathrm{~S}_{\lambda} * \mathrm{~T}_{\lambda} * \Delta \lambda}
$$

where $E_{\lambda}=$ relative erythemal spectral effectiveness, $S_{\lambda}=$ solar spectral irradiance, $T_{\lambda}=$ average spectral transmittance of the specimen, $\Delta \lambda=$ measured wavelength interval in nanometers $(\mathrm{nm})$, and $\lambda=$ wavelength of light in $\mathrm{nm}$. The percentage blocking of UV-A and UV-B is also calculated. This method requires that specimen UPF be measured in the warp (or wales), filling (or courses), and bias (45-degree rotation) directions, with the mean value of these measures reported.

A third document, ASTM D6603-00 Standard Guide for Labeling of UV-Protective Textiles, provides labeling requirements for textile products intended to protect human beings from UV-A and UV-B (ASTM, 2003). This document contains terminology for labeling UV-protective textiles. The labeling categories are "good" for UPF fabric values of at least 15 to 24, "very good" for UPF values of 25 to 39, and "excellent" for UPF values of 40 or higher.

Recently, the ISO-MET ${ }^{\circledR}$ UV-Meter was introduced as an al- ternative instrument for measuring UPF. This instrument is small, lightweight, and easily portable. It detects UV-B and UV$A$ in the ranges of $290 \mathrm{~nm}$ to $320 \mathrm{~nm}$ and $320 \mathrm{~nm}$ to $400 \mathrm{~nm}$, respectively (ISO-MET User's Manual, n.d.). Within 3 seconds of specimen placement, it provides a digital readout of UV-B, UV$A$, and UPF. In addition to being a convenient measuring device, this instrument can quickly demonstrate differences in fabric UPF to communicate with students or clients. However, no textile fabric research was identified that used this instrument. It was unknown whether the UVM would provide UPF calculations that were consistent with those measured by a SPM.

\section{Fabric Factors Affecting UPF}

Previous research has examined many factors affecting UVR transmission and the calculated fabric UPF. Fiber content, fabric structure, fabric weight, and porosity influence UVR transmission and fabric UPF. A few studies examined the effect of fiber content on UVR transmission (Crews et al., 1999; Davis et al. 1997; Gies, Roy, Toomey, \& McLennan, 1998). Polyester provides superior UV protection due to the fiber's benzene ring, if all other fabric features are held constant (Davis et al., 1997; Gies, Roy, Elliott, \& Zongli, 1994; Reinehr, Fuso, Hilfiker, $\&$ Schmidt, 1997). Although cotton is most used in summerweight clothing because of its absorbency and comfort, it is least effective in blocking UVR transmission.

Fabric construction (i.e., woven, knit, or nonwoven) also affects UVR transmission (Davis et al., 1997; Gies et al., 1994; Pailthorpe, 1998; Robson \& Diffey, 1990). The more tightly woven or knitted fabric, the less UVR transmits through the fabric. Fabric thickness and weight also were found to affect 
UVR transmission (Crews et al., 1999; Davis et al., 1997; Gies et al., 1994, 1998; Gies, Roy, McLennan, \& Toomey, 1997; Pailthorpe, 1998; Robson \& Diffey, 1990). Heavier fabrics have more fibers and yarns to penetrate compared with lighter ones; therefore, UVR is scattered and does not penetrate as directly to the skin. Fabric porosity is dependent on a combination of the attributes mentioned above but was identified as the main factor in UVR transmission by Crews et al. (1999), Reinehr et al. (1997), and Stanford et al. (1995a).

Fabric porosity is calculated by dividing the area of pores in the fabric by the total fabric area. The higher the fabric count or gauge, the more tightly woven or knitted the fabric; therefore, the lower the porosity when other fabric features are identical. UVR that passes directly through pores or holes in a fabric between yarns, for example, does not get scattered as it does when it strikes a fiber or yarn (Hilfiker, Kaufmann, Reinehr, \& Schmidt, 1996).

\section{Effects of Dyes and Laundering Additives on UPF}

Dyes, ultraviolet absorbers, and fluorescent whitening agents also can enhance UPF. Despite the common public perception that light-colored fabrics are cooler for summer, several research studies have demonstrated that dark colors offer greater sun protection, unless the light-colored fabrics are treated with ultraviolet absorbers or whitening agents. Dark colors produce higher UPF ratings because the concentration of dye in a textile affects UVR transmission. Investigations of dye effects include reports by Davis et al. (1997), Eckhardt and Rohwer (2000), Gies et al. (1994, 1997, 1998), Pailthrope (1994, 1998), and Reinehr et al. (1997). In a study primarily concerning the dye effect, Srinivasan and Gatewood (2000) re- ported that the "concentration and absorptivity of the dyes in the UV region" affected fabric UPF but that the color of a dye or fabric was not a reliable indicator of protection from UVR ( $p$. 41).

Most household detergents and many laundering additives contain fluorescent whitening agents (FWAs) or optical brightening agents (OBAs) that can increase fabric UPF during laundering. These compounds absorb UVR and re-emit it as visible light at the blue end of the spectrum (Hill \& Kolb, 2001). Studies documenting these effects include Eckhardt and Rohwer (2000), Hilfiker et al. (1996), Reinehr, Eckhardt, and Kaufmann (1996), Rohwer and Eckhardt (1998), and Zhou and Crews (1998). The research by Hilfiker et al. demonstrated an SPF increase through applying FWAs to cotton, silk, polyamide, and acrylic fiber fabrics.

UV-absorbing agents or UV-absorbers are colorless compounds that absorb UVR ranging from $290 \mathrm{~nm}$ to $400 \mathrm{~nm}$ (Pailthorpe, 1998). Studies showed that UV absorbing agents improve fabric UPF (Eckhardt \& Rohwer, 2000; Pailthorpe, 1998; Reinehr et al., 1997; Rohwer \& Eckhardt, 1998). Reinehr et al. (1997) reported UPF improvement for both natural and man-made fibers/fabrics finished with UV-absorbers.

\section{Effects of Repeated Laundering}

Repeated laundering with FWAs, OBAs, or UV-absorbers on UPF of knit and/or woven fabrics generally decrease UVR transmission, thus increasing UPF. Researchers investigated the effects of FWAs or OBAs (Eckhardt \& Rohwer, 2000; Stanford et al., 1995b; Zhou \& Crews, 1998) and effects of UV absorber (Eckhardt \& Rohwer, 2000; Rohwer \& Kvita, 1999; Zhou \& Crews, 1998). Eckhardt and Rohwer (2000) concluded that UV-ab- 
sorbers provided higher UPF values than FWAs after 5 through 20 cotton fabric launderings. Rohwer and Kvita (1999) applied UV absorber (Tinosorb ${ }^{\circledR} F R$ ) in rinse cycles, finding a substantial increase in UPF values after five washes of cotton fabrics. Zhou and Crews (1998) used the AATCC 1993 Standard Reference Detergent containing optical brightener in repeated laundering. They showed an increase in UPF value of cotton that was significantly greater for woven than knit fabrics. They reported no UPF improvement for nylon, Supplex ${ }^{\circledR}$ nylon, or polyester fabrics.

This investigation was designed to determine the effect of specimen orientation on UPF readings using two instruments, the effects of repeated laundering cycles using two household detergents-powdered Tide ${ }^{\circledR}$ with Bleach and Wisk ${ }^{\circledR}$ Liquidand two laundry additives-Rit ${ }^{\oplus}$ Whitener-and-Brightener and Rit $^{\oplus}$ SunGuard ${ }^{\mathrm{TM}}$ (hereafter, A, B, C, and D, respectively) on the UPF values of white, cotton jersey-knit and cotton/polyester blend pique-knit shirt fabrics. Hereafter, fabrics will be noted as cotton jersey and blended pique.

\section{NULL HYPOTHESES}

Based on the review of literature, the following null hypotheses were developed:

$\mathrm{H} 1$ : There is no difference in UPF results obtained from the UVM and SPM.

$\mathrm{H} 2$ : There is no significant difference in UPF related to the repeated measures from three different directions.

H3: There is no significant difference in UPF between cotton jersey and blended pique.

$\mathrm{H} 4$ : There is no significant difference in UPF related to five laundering levels.

H5: There is no significant difference in UPF related to four laundering treatments.

\section{METHOD}

\section{Experimental Design for Preparing Laundered Specimens} The laundered specimens were obtained using a $4 \times 5 \times 2$ factorial experimental design. The three independent variables (main effects) were (a) the type of laundry detergent/additives $(A, B, C$, and $D)$, (b) the number of launderings $(0,1,5,10$, and 15 ), and (c) the type of white knit fabrics (cotton jersey and blended pique). The dependent variable was the UPF value of knit fabric before and after each number of launderings with the detergent or laundry additive treatment.

\section{Materials}

Two types of knit shirts (eight of each) were purchased at a national chain discount store for use in this study. One type was an undershirt of $100 \%$ cotton jersey knit fabric (cotton jersey). The other was a polo style with pique-stitch knit fabric labeled as $60 \%$ cotton $/ 40 \%$ polyester (blended pique). Knit fabrics from control shirts that had not been laundered were examined to determine the fabric characteristics as shown in Table 1. All tests were conducted at standard atmospheric conditions. Fabric weight was determined using ASTM D3776 Fabric Weight/Mass per unit area of Woven Fabrics Option C: Small Swatch of Fabric, (ASTM, 2001b). Fabric thickness was conducted using ASTM D5729, Fabric Thickness of Materials/ Nonwoven Fabrics (ASTM, 2001c) using an Ames LG 2600-0- 
04 Dial micrometer with $10 \mathrm{~mm}$ presser foot. Fabric gauge was conducted using ASTM D3775-85 (ASTM, 2001d) with an Alfred Suter Thread Counter. Five replicate specimens were tested for weight and count; 10 were tested for thickness.

For laundry testing, each shirt was cut into quarters, with the first cut separating the shirt in half down the center front and center back, so there were a total of 16 blended-pique halves and 16 cotton jersey halves. Each half then was cut along underarm and shoulder seams so that there were 32 quarter-shirt pieces overall. Yardage of $100 \%$ cotton piquestitch knit was selected and cut into blocks of a size similar to the shirt pieces for use as dummy fabric to maintain the load size. The fiber content of the shirts and dummy fabric was confirmed by microscopic analysis. All shirt quarters (except one for each style shirt) were randomly assigned to one of the four detergent or additive treatments. The remaining shirt quarter pieces were retained and labeled as controls and received no laundering treatment.

The detergents and one additive used in this study were purchased at local discount stores, and their labels indicated that A, B, and C contained OBAs. These products were selected to represent those readily available to consumers; their manufacturers were not aware of our research. The additive $D$ was obtained from the manufacturer as it was being test marketed and contained $\mathrm{Ciba}^{\circledR}{ }^{\text {Tinosorb }^{T M}}$ FD, a UV-absorbing agent. Although it was provided to us at no charge, there was no input from the manufacturer concerning the conduct of this study. The amount of each product used for each treatment is also shown in Table 1.
TABLE 1: Summary of Materials Used

Part 1: Fabric Characteristics
Cotton Jersey $\quad$ Blended Pique

Fabric weight

$\begin{array}{lrlrl}(\mathrm{oz} / \mathrm{yd} 2) & 4.1 & (0.043) & 5.2 & (0.079) \\ (\mathrm{g} / \mathrm{m} 2) & 138.1 & (1.459) & 177.0 & (2.667)\end{array}$

Fabric thickness
(inch)
$0.017(0.0003)$
$0.031(0.001)$

Wale: Course: Wale: Course:

Fabric gauge

$$
\begin{aligned}
& \begin{array}{lllll}
\left(\text { yarns } / \text { in }^{2}\right) & 33.4 & 40.2 & 24.6 & 33.6
\end{array} \\
& \text { (0.548) (1.095) (0.548) (0.548) } \\
& \begin{array}{lll}
\text { (stitches/in } \left.{ }^{2}\right) & 73.6 & 58.2
\end{array}
\end{aligned}
$$

Part 2: Household Laundry Products Amount Used Per Treatment
A: powdered (g)
B: liquid ( $\mathrm{ml})$
C: brightener (g)
D: UV-absorber (g)

130

One package (28.4)

One package (28.4)

NOTE: Mean values are reported. Standard deviations are shown in parentheses.

\section{Laundering Procedures}

The laundering method used for the experiment was based on a modification of the AATCC Guidelines for Standardization of Home Laundry Test Conditions (AATCC, 1999). One modification was in the detergents used, and another was that we could not control temperatures precisely. A heavy-duty, extra-ca- 
pacity washer (Model A9900) and dryer (Model D9900), manufactured by the Maytag Company in Newton, lowa, were used. The washer was set on the regular setting for "white cotton sturdy" with hot water $\left(130^{\circ} \mathrm{F}\right), 10$ minutes washing time (total time with rinses of approximately 33 minutes), and maximum agitation. After the fabrics were placed in the washer, it was filled to a high water level of 15 gallons. The dryer was set on automatic drying for "regular fabrics," and shirt quarters were dried for approximately 45 minutes after each wash cycle.

The laundering process began with 30 shirt quarters ( 15 of each shirt type). After each laundry cycle (washing and drying), one quarter-shirt piece of each type shirt was removed randomly for UPF reading. Between washing loads, the washer was water-rinsed to take out the detergent residues from the tub. A piece of dummy fabric was added to the next laundering load to maintain the fabric-to-wash solution ratio in each succeeding cycle. Initially, a series of 15 washings was planned for each detergent and additive. However, this series was not completed for treatment $C$ or $D$ because the UPF readings of the fabric specimens were greater than 90 after 10 and 5 washes, respectively.

\section{Fabric Specimens for UPF Readings}

Four 2" $\times 2$ " fabric specimens were cut from each shirt quarter, for every treatment combination, to measure the UPF values. These were labeled with the number of launderings and type of laundry detergent or additive and then stored in zip-close bags. The direction of the wale was marked at the right-hand corner with the numeric label.

\section{UPF Measurement Instruments}

Two different UPF instruments were used to measure the UPF value of each specimen. The first instrument was ISO-MET ${ }^{\circledR}$ UV-Meter $^{\circledR}$ (UVM), which was loaned by $\mathrm{CIBA}^{\mathrm{TM}}$ to the researchers. The UVM provides digital readings of UPF value, UV $A$, and UV-B percentage transmission. The second instrument was a Cary UV-Visible spectrophotometer (SPM; model 93011297) with a UV light source and an integrating sphere to collect all the light transmitted through a fabric. The standard test method (AATCC Test Method 183-2000) with the SPM specifies fabric measurements from three directions because various fabric geometries give different patterns of shading that are direction related. But with the UVM, no standard test method exists. Our choice to compare outcomes based on the measuring direction was intended to determine whether such a recommendation should be made in the development of a standard protocol for the UVM. These instruments were located in separate laboratories at two midwestern universities. Measurements of the same specimens were taken independently to test the reliability of these two instruments.

\section{Procedures and Statistical Analysis}

Three replicate UPF measurements were taken from each of the four fabric specimens cut from each shirt quarter. These replicate measurements were from three directional orientations (i.e., wales, courses, bias), providing a total of 12 readings for each shirt quarter for each treatment (detergent or additive) at each laundering level $(0,1,5,10$, and 15). These readings were taken using both instruments. In statistical analysis, the different measuring orientations were treated as "repeated measures." The two sets of data obtained from the two meas- 
uring instruments were coded and entered for statistical analysis. Descriptive statistics were calculated. General linear model methods were used for estimating repeated measures analysis of variance models, multivariate tests were conducted, and reliability tests were performed to check for fulfillment of the key assumptions (in particular, homoscedasticity or equal variances) underlying the use of least squares models and to test the above hypotheses, using Statistical Package for Social Science (SPSS) version 10 statistical software (SPSS Inc., 1999).

Levene's test was performed to check for the equality of error variances between the repeated measures. The results were significant using both instruments except for one measurement $\left(F_{\text {Wale } 1}=6.45, p \leq .001 ; F_{\text {Course } 1}=6.58, p \leq .001 ; F_{\text {Bias } 1}=\right.$ $3.79, p \leq .001 ;$ FWale 2 $=2.67, p \leq .001$; FCourse $2=1.21, p=.232$; $\left.\mathrm{F}_{\text {Bias } 2}=2.45, p \leq .001\right)$. To correct this inequality of error variances, a natural logarithmic transformation was applied to the data.

\section{RESULTS}

\section{Descriptive Statistics}

Mean UPF values for both knit shirt fabrics increased significantly following repeated launderings, using each type of laundry detergent or additive. After the first laundering with detergents, mean UPF values increased significantly for both knit fabrics. With the cotton jersey, the mean UPF value increased significantly following repeated launderings using all four treatments, whether measured with the UVM or SPM (see Figure 1). The mean UPF values of both the cotton jersey knit and blended pique fabrics reached 50 as measured by the SPM after
10 launderings in either $\mathrm{A}$ or $\mathrm{B}-\mathrm{a}$ more than $300 \%$ increase as measured by the SPM.

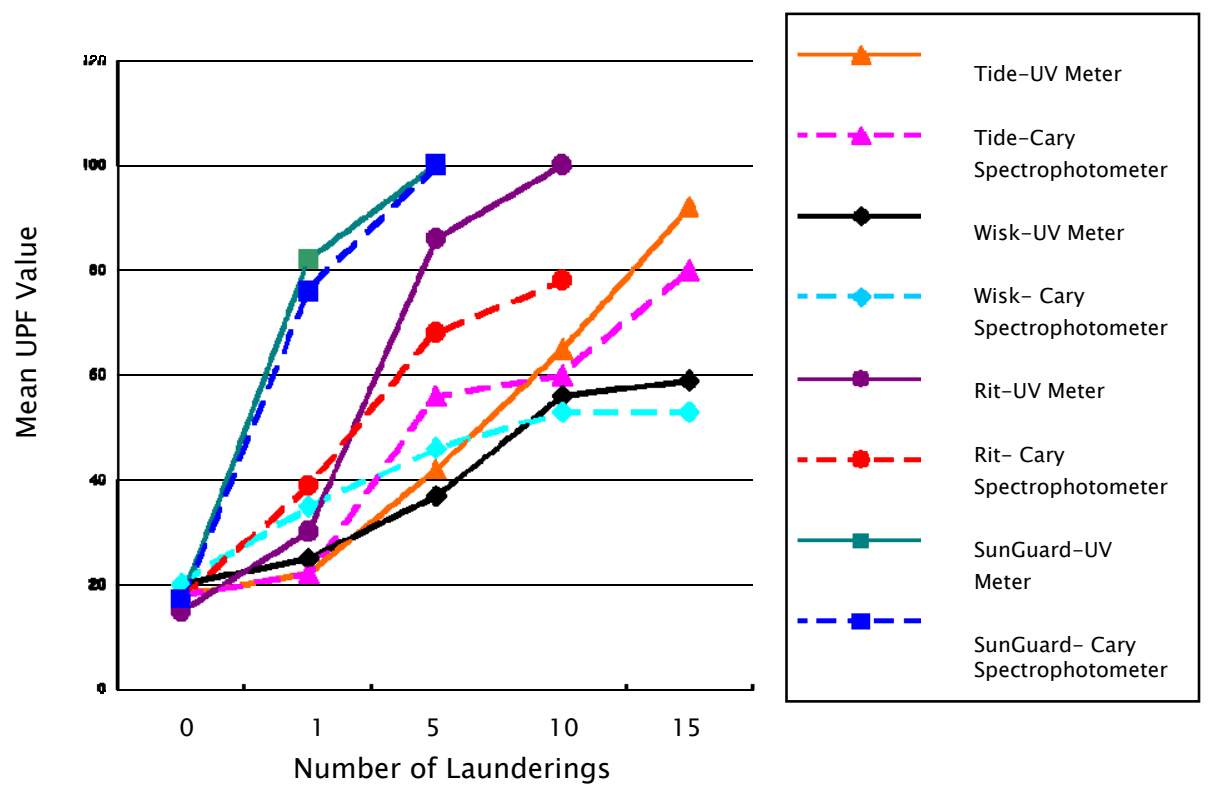

Figure 1: Mean UPF values of cotton jersey before and after laundering.

Use of the two laundry additives, C and D, also dramatically improved the mean UPF values for both fabrics. The UPF of the cotton knit after five launderings with $C$ was 68.3 (an increase of $506 \%$ as measured by the SPM). After one laundering with D, cotton jersey knit UPF.was 75.7 (a 561\% increase). These two laundry additives dramatically reduced the number of launderings needed to achieve a UPF value near 100, as compared with detergents $A$ and $B$. The mean UPF values for cotton fabric specimens steadily increased throughout the repeated launderings. With the blended pique, there was a higher mean UPF 
value before laundering than with the cotton jersey. The increase in mean UPF value for blended pique after laundering treatment was less dramatic than that for the cotton jersey, whether measured by the UVM or SPM (see Figure 2). This difference may be due to the shrinkage properties or fiber content of the fabrics. Usually cotton fabric shrinks more than blended fabric containing polyester. The increase in the mean UPF value of the blended pique provided a very acceptable level of protection because the UPF value was greater than 39 after 5 to 10 launderings using either detergent and after 5 launderings using the laundry additives $C$ and $D$. With both fabrics using C, a UPF of more than 50 was obtained with both instruments after 10 launderings; for $D$, a UPF of more than 50 was obtained with both instruments after 5 launderings. Therefore, launderings beyond this level were not conducted. In statistical model development, the empty cells thus created were treated as missing data.

\section{Hypotheses Testing Results}

To test Hypothesis 1, a within-sample validation between two different measuring instruments was conducted using the Cronbach's alpha measure of reliability. Means of UPF values on each fabric, for each type of laundry detergent or additive treatment before and after 1, 5, 10, and 15 launderings measured by the two instruments, are shown in Figures 1 and 2 . Hypotheses 2 through 5 were tested using repeated measures multivariate analysis of variance and post hoc analysis for pairwise comparisons of means using Scheffé multiple comparison tests.

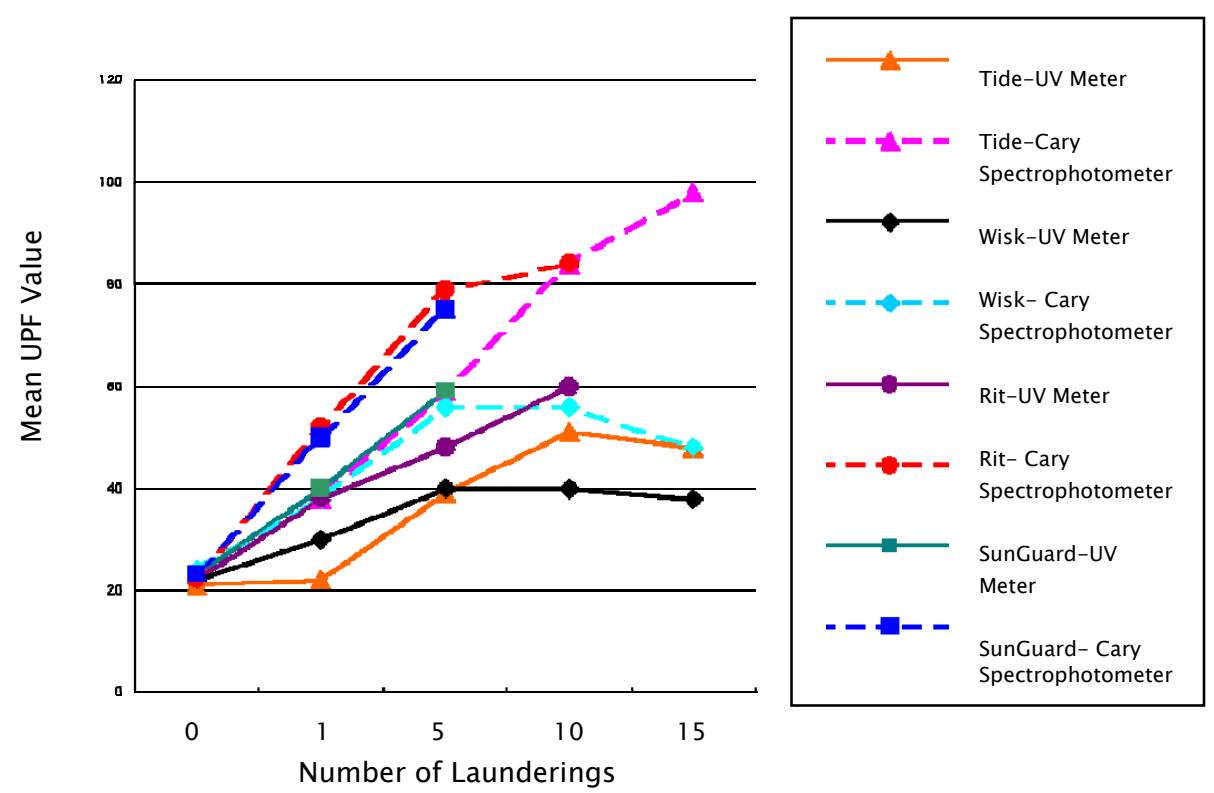

Figure 2: Mean UPF values of blended pique before and after laundering.

\section{Reliability Tests of Two UPF-Measuring Instruments ( $\mathrm{H} 1)$}

Reliability tests were conducted using Cronbach's alpha among UPF values obtained from three measuring directions and from two different measuring instruments. The reliability coefficient for the three measuring directions for the data obtained by UVM was .969 and for the data obtained by SPM, .993. The reliability of all six measures combined was .981 . These results show that both instruments indicated the similar direction of change in UPF. Therefore, Hypothesis 1 was supported. 


\section{Measuring Direction Effects ( $\mathrm{H} 2)$}

With regard to $\mathrm{H} 2$, the repeated measures analysis of variance (see Table 2) showed that there were no significant differences among mean UPF values found from measures obtained in the three different measuring directions using either UVM or SPM (see Tables 2 and 3). Thus, Hypothesis 2 was not rejected.

Repeated measures analysis of variance of effects in Tables 2 and 3 for both instruments also showed no significant interaction effects on UPF values related to other variables: measuring direction, detergent type, fabric type, and number of launderings. These results were confirmed by multivariate tests using Pillai's Trace, Wilks's Lambda, Hotelling's Trace, and Roy's Largest Root.

TABLE 2: Repeated Measures Analysis of Variance of Measuring Direction Effects of Instruments on UPF Based on the Results Obtained Using UV Meter

$\begin{array}{lrlcc}\text { Source } & \mathrm{df} & \mathrm{F} \text { Value } & \mathrm{p}>\mathrm{F} & \text { Partial Eta } \\ \text { Measuring direction (M) } & 2 & 0.96 & .384 & .009 \\ \mathrm{M} \times \text { Detergent (D) } & 6 & 1.57 & .158 & .044 \\ \mathrm{M} \times \text { Fabric (F) } & 2 & 0.50 & .607 & .005 \\ \mathrm{M} \times \text { Number of Launderings (L) } & 8 & 1.01 & .432 & .038 \\ \mathrm{M} \times \mathrm{D} \times \mathrm{F} & 6 & 1.27 & .272 & .036 \\ \mathrm{M} \times \mathrm{D} \times \mathrm{L} & 18 & 0.875 & .609 & .072 \\ \mathrm{M} \times \mathrm{F} \times \mathrm{L} & 8 & 1.29 & .251 & .048 \\ \mathrm{M} \times \mathrm{D} \times \mathrm{F} \times \mathrm{L} & 18 & 0.438 & .978 & .037 \\ \text { Error } & 204 & & & \end{array}$

NOTE: Partial Eta2 ${ }^{2}$ represents the variance in the dependent variable explained by each independent variable while holding the other independent variables constant.
TABLE 3: Repeated Measures Analysis of Variance of Measuring Direction Effects of Instruments on UPF Based on the Results Obtained Using Cary Spectrophotometer

\begin{tabular}{lrlrr} 
Source & df & F Value & $p>F$ & Partial Eta \\
\hline Measuring direction (M) & 2 & 0.118 & .888 & .001 \\
$M \times$ Detergent (D) & 6 & 1.09 & .368 & .031 \\
$M \times$ Fabric (F) & 2 & 1.67 & .190 & .016 \\
$M \times$ Number of Launderings (L) & 8 & 0.53 & .831 & .020 \\
$M \times D \times F$ & 6 & 2.13 & .052 & .059 \\
$M \times D \times L$ & 18 & 0.35 & .995 & .030 \\
$M \times F \times L$ & 8 & 1.15 & .330 & .043 \\
$M \times D \times F \times L$ & 18 & 0.71 & .797 & .059 \\
Error & 204 & & &
\end{tabular}

TABLE 4: Repeated Measures Analysis of Variance Testing for Effects on UPF Based on the Results Obtained Using UV Meter

\begin{tabular}{lrrrrrr} 
& \multicolumn{3}{c}{ Sum of } & \multicolumn{3}{c}{ Partial } \\
Source & df & Squares Squares & F Value & $\mathrm{p}>\mathrm{F}$ & $\mathrm{Eta}^{2}$ \\
\hline Detergent (D) & 3 & 2.208 & 0.736 & 108.88 & .000 & .762 \\
Fabrics (F) & 1 & 0.507 & 0.507 & 74.96 & .000 & .424 \\
Number of launderings (L) & 4 & 18.706 & 4.677 & 691.76 & .000 & .964 \\
$\mathrm{D} \times \mathrm{F}$ & 3 & 0.466 & 0.155 & 22.99 & .000 & .403 \\
$\mathrm{D} \times \mathrm{L}$ & 9 & 1.769 & 0.197 & 29.08 & .000 & .720 \\
$\mathrm{~F} \times \mathrm{L}$ & 4 & 2.734 & 0.683 & 101.10 & .000 & .799 \\
$\mathrm{D} \times \mathrm{F} \times \mathrm{L}$ & 9 & 0.553 & 0.061 & 9.09 & .000 & .445 \\
Error & 102 & .690 & .0067 & & &
\end{tabular}

\section{Fabric Effects (H3)}

Results of tests using UVM (see Table 4) indicated that there was a statistically significant difference between the UPF of cotton jersey and blended pique knit. Results of tests using 
SPM (see Table 5) also showed a statistically significant difference between cotton jersey and blended pique. Thus, Hypothesis 3 was rejected.

The predictive validity of fabric effects in the model results attained from using the data obtained from UVM (Eta2 $=.424)$ was much greater than the results attained using the data obtained from SPM (Eta2 $=.053)$. The effect of fabric type explained a moderate amount of total variance in the UPF values in the case of UVM; on the other hand, the effect of fabric type was significant but did not explain much about the total variance of UPF values obtained from SPM.

\section{TABLE 5: Repeated Measures Analysis of Variance Testing for Effects on UPF Based on the Results Obtained Using Cary Spec- trophotometer}

\begin{tabular}{lrrrrrrr} 
& \multicolumn{3}{c}{$\begin{array}{c}\text { Sum of } \\
\text { Source }\end{array}$} & \multicolumn{2}{c}{ Mean } & & \multicolumn{2}{c}{ Partial } \\
\hline Detergent (D) & 3 & 1.98 & 0.66 & 36.52 & .000 & .762 \\
Fabrics (F) & 1 & 0.10 & 0.10 & 5.69 & .019 & .053 \\
Number of launderings (L) & 4 & 20.87 & 5.22 & 288.19 & .000 & .919 \\
$\mathrm{D} \times \mathrm{F}$ & 3 & 1.09 & 0.36 & 20.13 & .000 & .372 \\
$\mathrm{D} \times \mathrm{L}$ & 9 & 2.58 & 0.29 & 15.85 & .000 & .583 \\
$\mathrm{~F} \times \mathrm{L}$ & 4 & 1.38 & 0.34 & 19.00 & .000 & .427 \\
$\mathrm{D} \times \mathrm{F} \times \mathrm{L}$ & 9 & 1.00 & 0.11 & 6.14 & .000 & .351 \\
Error & 102 & 1.85 & .018 & & &
\end{tabular}

\section{Repeated Laundering Effects $(\mathrm{H} 4)$}

Results using UPF values obtained by UVM (see Table 4) indicated a statistically significant difference in the mean UPF values obtained from the different levels of laundering treatments. Thus, Hypothesis 4 was rejected in the data from the
UVM. Table 6 shows post hoc tests, using Scheffé multiple comparisons that were conducted to show statistical differences in UPF values obtained with laundering levels.

Results using UPF values obtained by SPM (see Table 5) also showed a statistically significant difference in the mean UPF values obtained from the different levels of laundering treatments. Thus, Hypothesis 4 was rejected in the data from SPM. Results of Scheffé multiple comparisons are presented in Table 6 to show mean differences in UPF between before laundering and other laundering levels.

These results may be due to the missing data that occurred in the $C$ and $D$ treatments. For the $C$ treatment, researchers stopped after 10 launderings and for the D treatment, after 5 launderings, because those numbers of launderings produced UPF values higher than 50, a superior fabric rating for sun protection. Thus, Hypothesis 4 was rejected in the data from both UVM and SPM. $F$ values and partial $\mathrm{Eta}^{2}$ results indicated that repeated laundering effects alone explained the largest amount of total variation in UPF values $\left(\mathrm{Eta}^{2}=.964\right)$. This result provided strong evidence of the importance of the number of launderings with products containing OBAs or UV-absorbers in increasing the UPF values.

\section{Detergent/Laundry Additive Effects (H5)}

Results using UPF values obtained by UVM (see Table 4) indicated a statistically significant difference among laundry detergent or additive treatments. Thus, Hypothesis 5 was rejected by the data from the UVM. Again, Scheffé multiple comparisons are shown in Table 6 showing significant mean differences in UPF with the various treatments. The detergent effect alone explained the third-largest amount of total variance of the 
mean UPF value $\left(\mathrm{Eta}^{2}=.762\right)$.

Results using UPF values obtained by SPM (see Table 5) indicated a statistically significant difference among laundry detergents or additive treatments. Thus, Hypothesis 5 was rejected in the data obtained from SPM. Table 6, showing Scheffé multiple comparisons, revealed no significant mean differences among $\mathrm{A}, \mathrm{C}$, and $\mathrm{D}$. Treatment $\mathrm{C}$ provided lower UPF values than the other three. Hypothesis 5 , predicting no significant differences in UPF related to four laundering treatments, was rejected in the data from both UPF measuring instruments.

\section{Interaction Effects}

Table 4 shows the results of tests for interaction effects on mean UPF values obtained by UVM. Significant interactions were found among the three main effect variables-type of detergent/laundry additive, type of fabrics, and number of repeated launderings. All interaction effects were statistically significant $(p<.001)$, with partial effect size $\left(E_{t a}{ }^{2}\right)$ larger than .403 . These results indicate that interaction terms among and between all three independent variables showed significant differences in mean UPF values. In particular, the effect size of interaction effects of detergent and number of launderings $\left(\operatorname{Eta}^{2}=.720\right)$ and fabric and number of launderings $\left(\mathrm{Eta}^{2}=\right.$ .799) showed that those two interaction effects influence the mean UPF value. In Table 5, UPF values obtained by SPM identified significant interactions among type of detergent/laundry additive, types of fabric, and number of repeated launderings. All interaction effects were statistically significant $(p<.001)$, with partial effect sizes ranging from .372 to .583 . The predictive validity of the interactions in the SPM data was lower than those of the same interactions in the UVM.
TABLE 6: Scheffé Multiple Comparison Test Results

\begin{tabular}{|c|c|c|c|c|}
\hline \multirow{3}{*}{ Mean Differences Between: } & \multicolumn{4}{|c|}{ H4: Number of Launderings } \\
\hline & \multicolumn{2}{|c|}{ UVM } & \multicolumn{2}{|c|}{ SPM } \\
\hline & $\Delta \mathrm{x}$ & p Value & $\Delta \mathrm{x}$ & p Value \\
\hline 0 and 1 & -.288 & $<.001$ & -.316 & $<.001$ \\
\hline 0 and 5 & -.474 & $<.001$ & -.549 & $<.001$ \\
\hline 0 and 10 & -.530 & $<.001$ & -.531 & $<.001$ \\
\hline 0 and 15 & -.504 & $<.001$ & -.520 & $<.001$ \\
\hline 1 and 5 & -.187 & $<.001$ & -.233 & $<.001$ \\
\hline 1 and 10 & -.242 & $<.001$ & -.215 & $<.001$ \\
\hline 1 and 15 & -.217 & $<.001$ & -.204 & $<.001$ \\
\hline 5 and 10 & -.055 & $<.001$ & -.073 & $<.001$ \\
\hline 5 and 15 & -.030 & .379 & -.030 & .397 \\
\hline \multirow[t]{3}{*}{10 and 15} & .025 & .606 & .011 & .995 \\
\hline & \multicolumn{4}{|c|}{ H5: Types of Detergents/Laundry Additives } \\
\hline & \multicolumn{2}{|c|}{ UVM } & \multicolumn{2}{|c|}{ SPM } \\
\hline Mean Differences Between: & : $\Delta x$ & $\mathrm{p}$ Value & $\Delta \mathrm{x}$ & $\mathrm{p}$ Value \\
\hline$A$ and $B$ & .043 & $<.010$ & .060 & $\leq .010$ \\
\hline$A$ and $C$ & -.027 & .133 & -.018 & .148 \\
\hline$A$ and $D$ & -.049 & $<.010$ & -.032 & .352 \\
\hline B and C & -.070 & $<.001$ & -.055 & $<.050$ \\
\hline$B$ and D & -.092 & $<.001$ & -.102 & $<.001$ \\
\hline$C$ and D & -.022 & .396 & -.017 & .812 \\
\hline
\end{tabular}

NOTE: $A=$ Tide $^{\circledR} ; B=$ Wisk $^{\circledR} ; C=$ Rit $^{\circledR} ; D=$ SunGuard $^{\mathrm{TM}}$. 


\section{DISCUSSION}

Overall, the mean UPF values before and after launderings with detergents were higher than those found in other studies (Eckhardt \& Rohwer, 2000; Rohwer \& Kvita, 1999; Stanford et al., 1995a, 1995b; Zhou \& Crews, 1998). It is important to note that the initial UPF values of both fabrics used in this study were higher than in the cotton or cotton/polyester blend knits studied by Zhou and Crews (1998), who screened and selected fabrics that had no optical brighteners in them initially. Because the shirts used in this study were "off the rack" to simulate the consumer experience, whether they contained optical brighteners or not was unknown. It is interesting to compare the findings of this study in terms of percentage improvement in UPF with the use of OBAs in detergent, as compared to that reported by Zhou and Crews (1998). They reported a 295\% increase in UPF for cotton knit and $207 \%$ increase for PET/cotton blend knit. In this study, treatment A provided a $345 \%$ increase in UPF. This might be attributed to the differences in detergent formulations.

\section{Reliability of Two UPF-Measuring Instruments}

Gies, Roy, McLennan, Diffey, et al. (1997) reported significant differences in UPF results comparing SPM with the spectroradiometer but made no report concerning the UVM. We have shown that both the SPM and UVM have provided reproducible results, with Cronbach's alpha values higher than .98. Both instruments indicated the trend of increasing UPF based on repeated laundering.

\section{Measuring Direction Effects}

We found no significant difference in mean UPF values due to the measuring direction for the fabrics tested. Our results suggest that this idea should be tested with a wider range of fabric geometries to determine whether measuring direction-related differences can be identified in other structures. For the two knit fabrics used in this investigation, direction-related differences were not found.

\section{Fabric Effects}

Before laundering, the blended pique showed a higher mean UPF value than did the cotton jersey. This difference can be attributed to differences in fiber content, weight, and thickness. Polyester fiber absorbs more UVR because of its benzene ring, whereas cotton fiber is known to be transparent to UVR (Gies et al., 1994; Pailthorpe, 1998; Reinehr et al., 1997; Robson \& Diffey, 1990). The blended pique was heavier and thicker, with more than one set of comparatively larger yarns to shadow each other. The cotton jersey weighed less, was thinner, and had a higher count with smaller yarns close together, resulting in more but smaller interstices.

After laundering, the difference in UPF also can be explained partially by the differences in fabric weight and count. Although the blended pique fabric weighed more, it had the lower fabric gauge, with fewer yarns per square inch than that of cotton jersey, which would translate into higher porosity (larger interstices between yarns) compared to cotton jersey. The larger interstices would allow direct access for UV to strike the skin. The polyester content might help prevent shrinkage from laundering to close the interstices. Previous studies showed that cotton fabric shrinkage leads to a decrease in 
porosity (Crews et al., 1999; Reinehr et al., 1997; Stanford et al., 1995a); therefore, laundering of cotton jersey knit would assist in closing the already small interstices, thus boosting UPF values.

\section{Repeated Laundering Effects}

The mean UPF value increased significantly with 5 repeated launderings using each type of laundry detergent or additive. Both measuring instruments showed that the mean UPF values of both knit fabrics increased significantly after the first laundering with either of the four detergent or additive treatments. After 5 launderings with detergent, the mean UPF values for cotton fabrics increased with each level of laundering up to 15 washes. These results were consistent with those of Stanford et al. (1995a, 1995b), who found that UPF increased significantly following the first wash and remained high, for four out of five cotton shirts for up to 36 launderings.

After 5 repeated launderings with all four treatments, both fabrics attained the UPF value of 39 . These findings are consistent with Zhou and Crews (1998), who reported that use of optical brightener was one of the significant factors explaining an increase in UPF values. Our findings with regard to the effect of UV-absorbers are also consistent with previous research (Eckhardt \& Rohwer, 2000; Rohwer \& Kvita, 1999).

Both instruments showed a significant increase in UPF for the three main effects-fabric type, number of repeated launderings, and detergent/laundry additive type. Among those main effects, number of repeated launderings explained the largest amount of total variance of the increase of mean UPF values for both instruments. Detergent was the variable next in importance in explaining the mean UPF values. Both repeated laundering and detergent/additive effects are greater than that of fabric types on the increase of UPF values.

\section{Detergent/Laundry Additive Effects}

Scheffé multiple comparisons revealed that detergent type affected the mean UPF value. Compared with $B$, treatment A produced a higher UPF value. Two laundry additives, C and D, dramatically reduced the number of launderings required to achieve a UPF value near 100 , as compared with detergents $A$ and $B$. In addition, fabrics laundered with additives $C$ and $D$ showed significantly higher mean UPF values compared with those laundered with detergent B. For consumers, gaining the high UPF value rapidly with one wash, by using a product such as $\mathrm{D}$ to treat clothing, has distinct time-saving advantages.

\section{Future Research Directions}

The primary objectives of this study concerned the comparability of UPF results using the UVM and SPM and the effects of home laundering detergents and additives on UPF of inexpensive shirt fabrics. Several possibly useful variables were not evaluated because many were previously documented in literature (e.g., Stanford et al., 1995a). However, this study is not without limitations. We did not calculate percentage of shrinkage over the course of the 15 launderings because in the real world, consumers who launder new clothing cannot prevent either fabric shrinkage or OBA buildup from detergents. But from the theoretical viewpoint of textile science, it might be interesting to investigate carefully the proportion of change in UPF that might be attributed to shrinkage or to chemical treatment. Progressive shrinkage occurs with some fabrics on repeated laundering, which might affect porosity, thickness, 
and consequently UPF.

Our findings suggest other investigations beyond the scope of this article. For example, would the UVM also be a viable instrument to use as a substitute for the spectroradiometers in measuring fabric UPF? Would the findings for the UVM be comparable to the SPM over a broader range of fabric weights and types? Would other detergents and laundering additives offer as great an improvement in fabric UPF? Many other such questions invite researchers' attention.

\section{CONCLUSIONS AND IMPLICATIONS}

Through this research, we found that the UPF value of cotton and cotton/polyester blend knit fabric can be increased through home laundering by using commonly available household detergents and laundering additives with home laundering equipment. Whether the fabric UPF was measured by the UVM or SPM, a significantly higher mean UPF value was obtained after all laundering treatments in comparison with the control before laundering.

Both UVM and SPM provided reproducible results in estimating the fabric UPF. This result has important implications not only for textile testing but also for educational programs about sun safety. For example, the portable UVM could be used with confidence by land grant university extension specialists, educators, dermatologists, and other health care providers to teach about fabric UPF differences. The instrument is small, portable, and could easily be taken to classrooms or other informal settings to measure UPF transmitted by fabrics both with speed and acceptable accuracy. In this way, garments that consumers or students of any age are wearing could be tested quickly during educational programs to make a point and/or answer questions about how UV-protective they are.

We believe these improvements in fabric UPF also would occur for other summer-weight cotton or cotton-blend clothing as a result of laundering with detergents or additives containing OBAs or UV-absorbers. This finding has important implications for families. It means that probably most cotton or cotton-blend clothing laundered more than five times in ordinary detergent with OBAs will be more sun protective than when it was new. It also means that by using a product such as treatment $D$, new summer clothing for all family members can be rendered much more sun-protective with one washing. This effectively gives consumers great ability to gain increased sun protection via clothing. However, these findings, related to the ease of improvement of fabric UPF via launderings, do not negate the importance of clothing design and other measures for sun protection. A high fabric UPF in a garment that does not cover the skin is of little value in health terms. Therefore, it is of continued importance that educators work to improve consumer understanding of practices necessary to avoid excessive UVR exposure. Everyone should be aware that a high fabric UPF is affordable.

Implications for the apparel and textile industry are also suggested from our findings. First, apparel and textile firms may wish to adopt technologies to incorporate UV protection in fiber selection and/or during fabric finishing processes. Second, garment manufacturers should become aware of the importance of sun protection and encourage their suppliers to offer UPF-tested and -labeled fabrics for their use in product development. Third, retailers could include specially designed and UPF-labeled sun-protective clothing in their merchandise 
assortment for niche markets. By providing more UV-protective garments on the market at a reasonable price, the awareness of sun protection may be increased in the general population, which may eventually reduce incidence of UVR-related illness such as sunburn, premature aging of skin, skin cancer, and cataracts. Apparel producers and retailers can foster the improvement of understanding of sun-protective clothing and practices by partnering with educators to provide information for the public.

\section{REFERENCES}

American Association of Textile Chemists and Colorists (AATCC). (1999). Guidelines for standardization of home laundry test conditions. AATCC Technical Manual, 74, 383.

American Association of Textile Chemists and Colorists (AATCC). (2001). AATCC Test Method 183-2000 Transmittance or blocking of erythemally weighted ultraviolet radiation through fabrics. AATCC Technical Manual, 76, 349-351.

American Society for Testing and Materials (ASTM). (2003). D6603-00 Standard guide for labeling of UV protective textiles. Annual Book of ASTM Standards, 07.02, 1179-1183.

ASTM. (2001a).D 6544-00 Standard practice for preparation of textiles prior to UV transmission testing.

Annual Book of ASTM Standards, Textiles, 07.02, 1145-1148.

ASTM. (2001b).D3776-96 Standard test methods for mass per unit area (weight) of fabric. Annual Book of ASTM Standards, Textiles, 07.02, 79-82.
ASTM. (2001c).D5729-97 Standard test method for thickness of nonwoven fabrics. Annual Book of ASTM Standards, Textiles, 07.02, 821-824.

ASTM. (2001d). D3775-98 Standard test method for fabric count. Annual Book of ASTM Standards, Textiles, 07.02, 7678.

Capjack, L., Kerr, N., Davis, S., Fedosejevs, R., Hatch, K. L., \& Markee, N. L. (1994). Protection of humans from ultraviolet radiation through the use of textiles: A review. Family and Consumer Sciences Research Journal, 23(2), 198-218.

Crews, P. C., Kachman, S., \& Beyer, A. G. (1999). Influences on UVR transmission of undyed woven fabrics. Textile Chemist and Colorist, 31(6), 17-26.

Davis, S., Capjack, L., Kerr, N., \& Fedosejevs, R. (1997). Clothing as protection from ultraviolet radiation: Which fabric is most effective? International Journal of Dermatology, 36, 374379.

Eckhardt, H., \& Rohwer, C. (2000). Laundry additive for the sun protection of the skin. Sonderdruck aus SOFW-Journal, 124, 673-677.

Gies, H. P., Roy, C. R., Elliott, G., \& Zongli, W. (1994). Ultraviolet radiation protection factors for clothing. Health Physics, 56, 131-139.

Gies, H. P., Roy, C. R., McLennan, A., Diffey, B. L., Pailthorpe, M., Driscoll, C., et al. (1997).UV protection by clothing: An intercomparison of measurements and methods. Health Physics, 73(3), 456-464.

Gies, H. P., Roy, C. R., McLennan, A., \& Toomey, S. (1997). 
Clothing and protection against solar UVR. Journal of the Home Economics Institute of Australia, 5(2), 2-6.

Gies, H. P., Roy, C. R., Toomey, S., \& McLennan, A. (1998). Protection against solar ultraviolet radiation. Mutation $R e-$ search, 422, 15-22.

Hilfiker, R., Kaufmann, W., Reinert, G.,\& Schmidt, E. (1996). Improving sun protection factors of fabrics by applying UVabsorbers. Textiles Research Journal, 66(2), 61-70.

Hill, J. W., \& Kolb, D. K. (2001). Chemistry for changing times (9th ed.). Upper Saddle River, NJ: Prentice Hall.

ISO-MET user's manual, UV-2 protection meter. (n.d.). Geneva, Switzerland: ISO-MET.

L. L. Bean, Inc. (2003). Clothing for men. The sunblock you wear. Retrieved February 5, 2003, from http://www.Ilbean.com/shop/shopByCatalog/UY/p32uy.html

Pailthorpe,M.(1994).Textiles and sun protection: The current situation. Australian Textiles, 14(6), 54-66.

Pailthorpe, M. (1998). Apparel textiles and sun protection: A marketing opportunity or a quality control nightmare? $\mathrm{Mu}$ tation Research, 422, 175-183.

Reinehr, D., Eckhardt, C., \& Kaufmann, W. (1996, March). Skin protection against ultraviolet light by cotton textiles treated with optical brighteners (pp. 264-276). Proceedings of World Surfactants Congress 4th, Barcelona, Spain.

Reinert, G., Fuso, F., Hilfiker, R., \& Schmidt, E. (1997).UV-protecting properties of textile fabrics and their improvement. Textile Chemist and Colorist, 29(12), 36-43.
Robson, J., \& Diffey, B. (1990). Textiles and sun protection. Photodermatology, Photoimmunology and Photomedicine, 7, 32-34.

Rohwer, H., \& Eckhardt, C. (1998). Laundry additive for the sun protection of the skin. Sonderdruck aus SOFW-Journal, 124, $1-4$.

Rohwer, H., \& Kvita, P. (1999). Sun protection of the skin with a novel UV absorber for rinse cycle application. Sonderdruck aus SOFW-Journal, 125, 1-5.

SPSS, Inc. (1999). SPSS $₫$ base 10.0 user guide. Chicago: Author.

Srinivasan, M., \& Gatewood, B. M. (2000). Relationship of dye characteristics to UV protection provided by cotton fabric. Textile Chemist and Colorist, 32(4), 36-43.

Stanford, D. G., Georgouras, K. E., \& Pailthorpe, M. T. (1995a). Sun protection by a summer-weight garment: The effect of washing and wearing. Medical Journal of Australia, 162, 422425.

Stanford, D. G., Georgouras, K. E., \& Pailthorpe, M. T. (1 995b). The effect of laundering on the sun protection afforded by a summer weight garment. Journal of the European Academy of Dermatology and Venereology, 5, 28-30.

Wang, S. Q., Kopf, A. W., Marx, J., Bogdan, A., Polsky, D., \& Bart, R. S. (2001). Reduction of ultraviolet transmission through cotton T-shirt fabrics with low ultraviolet protection by various laundering methods and dyeing: Clinical implications. Journal of American Academy of Dermatology, 44(5), 767-774.

Zhou, Y., \& Crews, P. C. (1998). Effect of OBAs and repeated 
Kim, Stone, Crews, Shelley, Hatch

launderings on UVR transmission through fabrics. Textile Chemist and Colorist, 30(1 1), 19-24.

Authors' Note: This article was financially supported by the lowa Agriculture and Home Economics Experiment Station, Ames, lowa, Project No. 2599 with Hatch Act and state of lowa funds, lowa State University Extension, and the University of Nebraska-Lincoln Agricultural Research Division, Lincoln, NE 68583 (Journal Series No. 14090). The work was conducted as part of the USDA NC-170 Regional Research Project.

Family and Consumer Sciences Research Journal, Vol. 33, No. 2, December 2004, pp. 141-158

DOI: $10.1177 / 1077727 \times 04269185$

(c) 2004 American Association of Family and Consumer Sciences 